\title{
Signals of Dark Matter in hypercolor vectorlike extension of the SM
}

\author{
Vitaly Beylin ${ }^{1, *}$, Maxim Bezuglov ${ }^{1,2,3, * *}$, and Egor Tretiakov ${ }^{1,2,3, * * *}$ \\ ${ }^{1}$ Southern Federal University, Institute of Physics \\ ${ }^{2}$ Moscow Institute of Physics and Technology \\ ${ }^{3}$ Joint Institute of Nuclear Researches, Bogoliubov Laboratory of Theoretical Physics
}

\begin{abstract}
In the framework of hypercolor extension of the Standard Model we consider cosmic rays scattering off hidden mass candidates. Specifically, there are two components of the Dark Matter in this model having close masses but different origin. As a result, neutrino production in the processes of scattering of high energy electrons on these DM candidates is substantially different, and the secondary neutrino can be seen, in principle, at neutrino detectors. We also note other interesting aspects of these type reactions.
\end{abstract}

\section{Introduction}

The discovery of the Higgs light boson has shifted the interest and activity of physicists to the analysis of the effects predicted in numerous variants of the Standard Model (SM) extension. Along with the refinement of the parameters of known SM particles, the study of the details of their production and decay, an origin of both the energy scales of new physics (at which new degrees of freedom are manifested themselves) and type of dynamic symmetries is unclear.

Therefore, it is necessary to lay new paths in uncharted territory relying on analogies, conjectures, and intuition. At the same time, it is difficult to expect help and hints from the results obtained by the LHC collaborations - despite the painstaking and continuous advancement of the front towards ever higher energies, there are no clear indications of the new physics effects. The search for such manifestations, however, is necessary, since the SM is clearly an open model leaving a number of important issues unresolved. In particular, this is a question about the origin, structure and measurement of the hidden mass characteristics and distribution in the Universe. Obviously, the absence of direct evidence of the Dark Matter (DM) nature means a special role in the analysis of the entire set of indirect data (multi-messenger approach) on processes with the possible participation of the DM particles.

So, all possible astrophysical data from galactic and extragalactic sources should be gathered and carefully considered to select possible signals of the DM. Moreover, the masses of $\mathrm{DM}$ candidates are in a very broad interval - from $\mathrm{eV}$ to dozens of $\mathrm{TeV}$ - and, therefore, there is need in Earth and space observatories which are able to measure spectra of photons, neutrino, leptons, protons and nuclei with sufficient accuracy and in various energy diapasons. From the other side, there should be analyzed possible manifestations of the DM candidates

\footnotetext{
*e-mail: vitbeylin@ gmail.com

**e-mail: bezuglov.ma@phestech.edu

***e-mail: tretiakov.ea@phystech.edu
} 
from various SM extensions. For example, some additional chance to catch the DM particle "by the tail" can be presented by consideration of the cosmic ray scattering off the DM clusters.

Here, we present some results on inelastic interaction of high energy electron component of cosmic rays with the hidden mass particles. More exactly, we consider two-component dark matter which follows from hypercolor extension of the SM; in this model, two types of neutral stable pseudo-Nambu-Goldstone (pNG) states emerge connecting with different hyper-quark currents.

In the first Section some details of the model structure are presented, in the second Section we give estimations of necessary parameters - masses, mixing angle etc. Third Section contains our results on the inelastic scattering of cosmic electrons off the DM particles. We also discuss some features of these processes in Conclusion.

\section{Hypercolor extension of the SM in brief}

Hypercolor model extends the number of fermion degrees of freedom in the SM by adding doublet(s) of heavy H-quarks in confinement analogously to initial Technicolor [1, 2]. However, some problems of the TC can be eliminated due to transformation of two initial left $\mathrm{H}$-quark doublets into one doublet of Dirac states interacting with standard bosons as vector current [3]. Hypercharges of H-quarks are equal and opposite in sign - then anomalies in the model are absent. H-quarks also are degenerated in mass. The next step in the model construction is the transition to physical fields (constituent quarks) using ideas of linear sigmamodel. Then, it appears an additional scalar state, hyper- $\sigma-$ meson which is mixed with the standard Higgs boson. Breaking of the global symmetry, $S U(4) \rightarrow S p(4)$, results in a number of pNG states involving triplet of hyper-pions and hyper-baryon states; these fields are supposed as low-lying ones. Most important, specific symmetry of the Lagrangian provides stability of neutral $\mathrm{H}$-pion (due to modified charge conjugation parity) and $\mathrm{H}$-baryons (H-diquarks) and $B^{0}$ and $\bar{B}^{0}$ as a consequence of hyper-baryon number conservation $[4,5]$.

All important parts of the model Lagrangian are written in [5, 6]; here, only Lagrangian of scalars self-interactions is presented for demonstration of some specific channels of interaction.

$$
\begin{gathered}
L=-\lambda_{00} h^{3}\left(v+\frac{1}{4} h\right)-\frac{1}{4} \lambda_{11}\left(B_{\dot{\alpha}} B_{\dot{\alpha}}+\sigma^{\prime 2}\right)\left(B_{\dot{\alpha}} B_{\dot{\alpha}}+\sigma^{\prime 2}+4 u \sigma^{\prime}\right) \\
-\frac{1}{4} \lambda_{01} h\left[(2 v+h)\left(B_{\dot{\alpha}} B_{\dot{\alpha}}+\sigma^{\prime 2}\right)+2 u \sigma^{\prime} h\right]-\frac{1}{4} \lambda_{02} h(2 v+h)\left(A_{\dot{\alpha}} A_{\dot{\alpha}}+\tilde{\eta}^{2}\right) \\
-\frac{1}{4} \lambda_{12}\left(B_{\dot{\alpha}} B_{\dot{\alpha}}+\sigma^{\prime 2}+2 u \sigma^{\prime}\right)\left(A_{\dot{\alpha}} A_{\dot{\alpha}}+\tilde{\eta}^{2}\right)-\frac{1}{4} \lambda_{22}\left(A_{\dot{\alpha}} A_{\dot{\alpha}}+\tilde{\eta}^{2}\right)^{2} \\
-\lambda_{33}\left[-\left(u+\sigma^{\prime}\right) \tilde{\eta}+\tilde{a}_{k} \tilde{\pi}_{k}+\bar{B}^{0} A^{0}+\bar{A}^{0} B^{0}\right]^{2}
\end{gathered}
$$

where $A_{\dot{\alpha}} A_{\dot{\alpha}}=2 \tilde{a}^{+} \tilde{a}^{-}+\tilde{a}^{0} \tilde{a}^{0}+2 \bar{A}^{0} A^{0}, B_{\dot{\alpha}} B_{\dot{\alpha}}=2 \tilde{\pi}^{+} \tilde{\pi}^{-}+\tilde{\pi}^{0} \tilde{\pi}^{0}+2 \bar{B}^{0} B^{0}$.

Note also, an analysis of Peskin-Tackeuchi parameters results in some constraints on the mixing of Higgs boson and $\sigma-$ meson, to avoid discrepancies with T-parameter value and SM Higgs boson characteristics it should be $\sin \theta \lesssim 0.1[2,7]$. So, to study reactions with the DM candidates participation, it sufficiently to know masses of the DM components (neutral H-pion and H-baryon), mass and v.e.v. of $\tilde{\sigma}-$ meson and the angle of mixing.

As for masses of H-pions, it is well known that mass difference is $\Delta M_{\tilde{\pi}}=m_{\tilde{\pi}^{ \pm}}-m_{\tilde{\pi}^{0}} \approx$ $0.16 \mathrm{GeV}[8,9]$, and charged hyper-pions are unstable[5]. Nevertheless, despite of violation 
of isotopic invariance by non-zero mass splitting, hyper parity remains conserved, so the neutral H-pion is stable even with an account of higher order corrections.

However, $\tilde{\pi}^{0}$ and $B^{0}$ masses are equal at the tree level with the non-zero mass difference followed from electroweak insertions $\Delta M_{B \tilde{\pi}}=m_{B^{0}}-m_{\tilde{\pi}^{0}}$ :

$$
\begin{aligned}
\Delta M_{B \tilde{\pi}}= & \frac{-g_{2}^{2} m_{\tilde{\pi}}}{16 \pi^{2}}\left[8 \beta^{2}-1-\left(4 \beta^{2}-1\right) \ln \frac{m_{\tilde{\pi}}^{2}}{\mu^{2}}+2 \frac{M_{W}^{2}}{m_{\tilde{\pi}}^{2}}\left(\ln \frac{M_{W}^{2}}{\mu^{2}}-\beta^{2} \ln \frac{M_{W}^{2}}{m_{\tilde{\pi}}^{2}}\right)\right. \\
& \left.-8 \frac{M_{W}}{m_{\tilde{\pi}}} \beta^{3}\left(\arctan \frac{M_{W}}{2 m_{\tilde{\pi}} \beta}+\arctan \frac{2 m_{\tilde{\pi}}^{2}-M_{W}^{2}}{2 m_{\tilde{\pi}} M_{W} \beta}\right)\right],
\end{aligned}
$$

where $\beta=\sqrt{1-\frac{M_{W}^{2}}{4 m_{\tilde{\pi}}^{2}}}$. The value of $\Delta M_{B \tilde{\pi}}$ depends on a renormalization point because of origin of these pNG states from different H-quark currents. So, the value of renormalization scale should be added to other necessary input parameters.

\section{Phenomenology of hypercolor: masses, mixing, etc.}

Having mass splittings for stable components of hidden mass, we suppose later that $\Delta M_{B \tilde{\pi}}$ and $\Delta M_{\tilde{\pi}}$ are small in comparison with $M_{B}$ and $M_{\tilde{\pi}}$. Then, to consider kinetics of the DM burn-out five Boltzmann equations for two states of the neutral H-baryon, $B^{0}, \bar{B}^{0}$ and two charged H-pions together with the neutral one are needed. It means that processes of coannihilation[10] should contribute to the annihilation cross section (see also[9]).

So, we start from the system of five basic Boltzmann equations[6] for $\tilde{\pi}^{+}, \tilde{\pi}^{-}, \tilde{\pi}^{0} ; \mu, v=$ $B, \bar{B}$, which can be rewritten as the system of equations for densities of particles, $n_{\tilde{\pi}}=\sum_{i} n_{i}$ and $n_{B}=\sum_{\mu} n_{\mu}$, also an equilibrium particle density, $n_{e q}$, is introduced. So, we have:

$$
\begin{aligned}
\frac{d a^{3} n_{\pi}}{a^{3} d t}= & <\bar{\sigma} v>_{\tilde{\pi}}\left(n_{\tilde{\pi}}^{2}-\left(n_{\tilde{\pi}}^{e q}\right)^{2}\right)-<\sigma v>_{\tilde{\pi} \tilde{\pi}}\left(n_{\tilde{\pi}}^{2}-\frac{\left(n_{\tilde{\pi}}^{e q}\right)^{2}}{\left(n_{B}^{e q}\right)^{2}} n_{B}^{2}\right)+ \\
& <\sigma v>_{B B}\left(n_{B}^{2}-\frac{\left(n_{B}^{e q}\right)^{2}}{\left(n_{\tilde{\pi}}^{e q}\right)^{2}} n_{\tilde{\pi}}^{2}\right), \\
\frac{d a^{3} n_{B}}{a^{3} d t}= & <\bar{\sigma} v>_{B}\left(n_{B}^{2}-\left(n_{B}^{e q}\right)^{2}\right)+<\sigma v>_{\tilde{\pi} \tilde{\pi}}\left(n_{\tilde{\pi}}^{2}-\frac{\left(n_{\tilde{\pi}}^{e q}\right)^{2}}{\left(n_{B}^{e q}\right)^{2}} n_{B}^{2}\right)- \\
& <\sigma v>_{B B}\left(n_{B}^{2}-\frac{\left(n_{B}^{e q}\right)^{2}}{\left(n_{\tilde{\pi}}^{e q}\right)^{2}} n_{\tilde{\pi}}^{2}\right)
\end{aligned}
$$

where:

$$
\begin{aligned}
& <\bar{\sigma} v>_{\tilde{\pi}}=\frac{1}{9} \sum_{i, j}<\sigma v>_{i j}, \quad\left\langle\bar{\sigma} v>_{B}=\frac{1}{4} \sum_{\mu, v}<\sigma v>_{\mu v}\right. \\
& <\sigma v>_{\tilde{\pi} \tilde{\pi}}=\frac{1}{9}\left(<\sigma v>\left(\tilde{\pi}^{0} \tilde{\pi}^{0} \rightarrow B \bar{B}\right)+2<\sigma v>\left(\tilde{\pi}^{+} \tilde{\pi}^{-} \rightarrow B \bar{B}\right)\right) \\
& <\sigma v>_{B B}=\frac{1}{2}\left(<\sigma v>\left(B \bar{B} \rightarrow \tilde{\pi}^{0} \tilde{\pi}^{0}\right)+<\sigma v>\left(B \bar{B} \rightarrow \tilde{\pi}^{-} \tilde{\pi}^{+}\right)\right)
\end{aligned}
$$


In the case considered, $m_{\tilde{\pi}} / M_{B} \approx 1$, so $n_{B}^{e q} / n_{\tilde{\pi}}^{e q}=2 / 3$, then the system is simplified. Besides, we use approximation $\Delta M_{B^{0} \tilde{\pi}^{0}} \mid / m_{\tilde{\pi}^{0}} \lesssim 0.02$ when the cross sections $\left\langle\sigma v>_{\tilde{\pi} \tilde{\pi}}\right.$ and $\langle\sigma v\rangle_{B B}$ should be presented in the form (as it takes place for any processes near the threshold[10]):

$$
<\sigma v>_{B B} \approx<\left(a+b v^{2}\right) v_{2}>=\frac{2}{\sqrt{\pi} x}\left(a+\frac{8 b}{x}\right),
$$

where $x=m_{\tilde{\pi}} / T$ and $v_{2}$ is the velocity of final particles in the center-of-mass frame. This system is solved numerically with the notation: $Y=n / s$, and then $W=\log Y$ ( $s$ is the entropy density). Solutions can be seen as some regions in the plane H-pion and H-sigma masses for various values of renormalization scale $\mu$ and v.e.v. $u$. Note, these masses are connected by some exact relation in the case of zero mixing [5].

As a result (see Fig. 1), there are three possible regions to explain the recent DM density by the vectorlike H-color model, for more detail see [6]:

Region 1: $M_{\tilde{\sigma}}>2 m_{\tilde{\pi}^{0}}$ and $u \geq M_{\tilde{\sigma}}$-a significant H-pion fraction is possible at small mixing and large masses of H-pions;

Region 2: small fraction of H-pions with $m_{\tilde{\pi}} \approx 300-600 \mathrm{GeV}$ for practically the same values of $M_{\tilde{\sigma}}, m_{\tilde{\pi}^{0}}, u$;

Region 3: $M_{\tilde{\sigma}}<2 m_{\tilde{\pi}}$, H-pion fraction can be large for small mixing and large $m_{\tilde{\pi}^{0}}$.

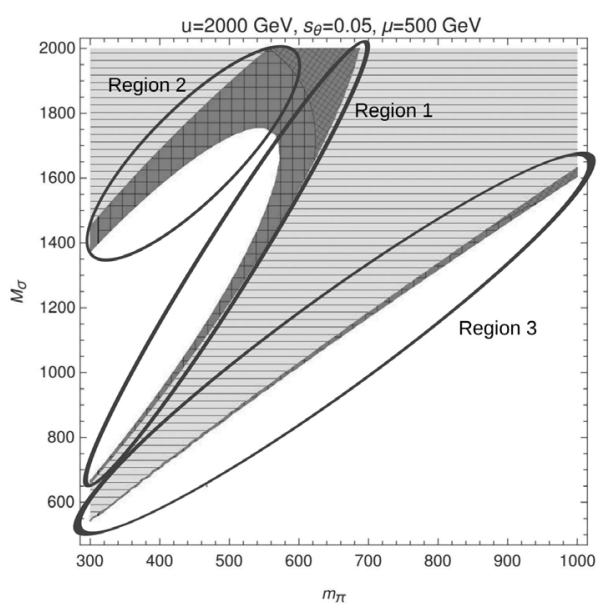

Figure 1. Numerical solution of the kinetic equations system in a phase diagram in terms of $M_{\tilde{\sigma}}$ and $m_{\tilde{\pi}}$ parameters.

Here, the hatching with vertical cells denotes areas with reasonable DM relic density with H-pion fraction less than 25 percents $\left(0.1047 \leq \Omega h_{H P}^{2}+\Omega h_{H B}^{2} \leq 0.1228\right.$ and $\Omega h_{H P}^{2} /\left(\Omega h_{H P}^{2}+\right.$ $\left.\left.\Omega h_{H B}^{2}\right) \leq 0.25\right)$. The hatching with oblique cells indicates region with the same values of parameters but pion fraction is $\left(0.1047 \leq \Omega h_{H P}^{2}+\Omega h_{H B}^{2} \leq 0.1228\right.$ and $0.25 \leq \Omega h_{H P}^{2} /\left(\Omega h_{H P}^{2}+\right.$ $\Omega h_{H B}^{2} \leq 0.4$ ). Note, there are no regions where H-pion component dominates in the DM. It is resulted from tree-level interaction of $\mathrm{H}$-pions with weak vector bosons; $B^{0}-$ component interact with ordinary quarks and leptons via $\mathrm{H}$-quark and $\mathrm{H}$-pion loops, an important channel is via scalars - Higgs-boson and $\sigma-$ meson.

Hatching with horizontal lines corresponds to permitted regions $\left(\Omega h_{H P}^{2}+\Omega h_{H B}^{2} \leq 0.1047\right)$, here we have not correct value for the DM abundance in the H-color model. Hatching with vertical lines are forbidden by XENON experiments[11, 12]. 
Now, for the next step we can use for the DM candidates masses the values 600, 800 and $1200 \mathrm{GeV}$. Correspondingly, we can use for $m_{\sigma}=1000-1600 \mathrm{GeV}$.

\section{Scattering of high-energy cosmic rays off the Dark Matter}

The importance of indirect DM searches follows from the absence of direct signals of new physics from collider and underground detectors. So, indirect DM effects should be analyzed carefully, these manifestations are: the DM annihilation into photons, generation of highenergy photons in cosmic rays scattering off the DM, virtual internal bremsstrahlung in the DM annihilation, lepton pairs production in the unstable DM decay, and also neutrino (lepton) creation in cosmic rays scattering. The last process is a perspective part of multi-messenger study of the DM origin, structure and distribution in the Galaxy. Considering interaction of cosmic leptons with the DM particles, we have two different contributions due to two components of the DM. First, electrons with energies up to $\sim 10 \mathrm{TeV}$ can produce neutrino in the reaction $e \tilde{\pi}^{0} \rightarrow v_{e} \tilde{\pi}^{-}$, here, charged H-pion $\tilde{\pi}^{-}$will decay with $\operatorname{Br}\left(\tilde{\pi}^{-} \rightarrow \tilde{\pi}^{0} e v_{e}^{\prime}\right) \approx 0.01$ and $\operatorname{Br}\left(\tilde{\pi}^{-} \rightarrow \tilde{\pi}^{0} \pi^{-}\right) \approx 0.99$ [5]. This channel is resulted from interaction of H-pions with vector mesons, namely, electron produce the secondary $v_{e}$ due to $W e v_{e}-$ vertex together with soft secondaries $e^{\prime} v_{e}^{\prime}$ or $\mu v_{\mu}$ which are generated by $\tilde{\pi}^{-}$decay[6].

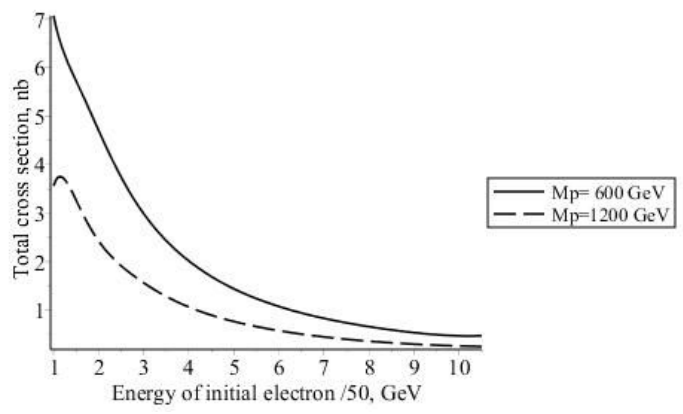

Figure 2. Total cross section of the process in dependence on the initial electron energy. For different final channels corresponding branchings should be used.

The cross section of the reaction $e \tilde{\pi}^{0} \rightarrow v_{e} \tilde{\pi}^{-}$has the form:

$$
d \sigma\left(e \tilde{\pi}^{0} \rightarrow v_{e} \tilde{\pi}^{-}\right)=\pi \frac{\alpha^{2} \cdot(1+\cos \theta) d \cos \theta}{E_{e}^{2} \alpha_{e}} \cdot \frac{f_{1}\left(\alpha_{e}, \cos \theta\right)}{f_{2}\left(\alpha_{e}, \alpha_{W}, \cos \theta\right)},
$$

where $\alpha_{e}=E_{e} / m_{\tilde{\pi}^{0}}, \alpha_{W}=E_{e} / M_{W}$, energy of incoming electron and masses of neutral H-pion and W-boson are $E_{e}, \quad m_{\tilde{\pi}^{0}}, \quad M_{W}$, correspondingly. Also, we get

$$
\begin{gathered}
f_{1}\left(\alpha_{e}, \cos \theta\right)=\left[1-\frac{2 \cos \theta}{1+\alpha_{e}(1-\cos \theta)}+\frac{1}{\left(1+\alpha_{e}(1-\cos \theta)\right)^{2}}\right]^{1 / 2}, \\
f_{2}\left(\alpha_{e}, \alpha_{W}, \cos \theta\right)=\left[\frac{1}{\alpha_{W}^{2}}+\frac{2(1-\cos \theta)}{1+\alpha_{e}(1-\cos \theta)}\right]^{2} .
\end{gathered}
$$

Due to analysis of kinetics, following values can be used: $m_{\tilde{\pi}^{0}}=600 \mathrm{GeV}$ and $1200 \mathrm{GeV}$ being in agreement with the collider restrictions(see [13] and references therein). We also suppose that the second final neutrino, $v_{l}^{\prime}$, is soft in comparison with $v_{e}$ produced by $e v W$ 


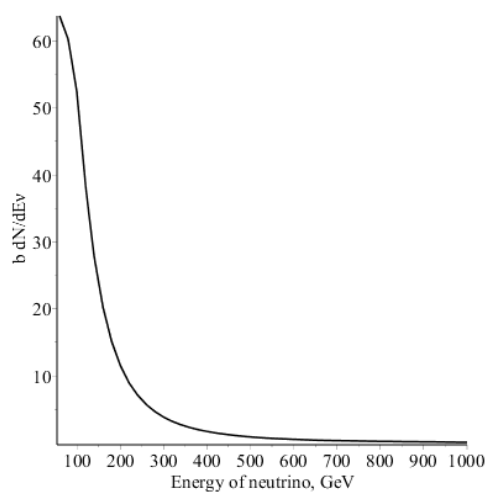

Figure 3. Differential flux of secondary neutrino for incident electron energies from 50 up to $1000 \mathrm{GeV}$, H-pion mass $m_{\tilde{\pi}}=1200 \mathrm{GeV}$, coefficient $b=k_{f} \cdot 10^{7} E_{v}^{2}, \mathrm{GeV} / \mathrm{cm}^{2} \cdot s \cdot s r, k_{f}=1$ with amplifying factor $f$ and $k_{f} \approx 0.14$ without the factor.

vertex. At initial electron energy $E_{e}=(100-1000) \mathrm{GeV}$ we get (see Fig. 2) the cross section varies from $O(10) \mathrm{nb}$ to $O(0.1) \mathrm{nb}$ and has maximum in forward direction.

Neutrino flux is calculated in the framework of known procedure[14]:

$$
d N / d E_{v}=r_{\odot} \rho_{\odot} \bar{J} \frac{1}{M_{\tilde{\pi}^{0}}} \int d \Omega_{v} \int d E_{e} \frac{d \Phi}{d E_{e}} \frac{d^{2} \sigma}{d \Omega_{v} d E_{v}},
$$

where the DM density is $\rho_{\odot}=0.3 \mathrm{GeV} / \mathrm{cm}^{3}$ at the Sun location and $r_{\odot}=8.33 \mathrm{kpc}$. The spectrum of cosmic electrons can be approximated as $d \Phi / d E_{e}=k_{e}(E / G e V)^{-a}$ with $a=3.15$ [15] and $k_{e}=0.01 \mathrm{GeV}^{-1} \mathrm{~cm}^{-2} \mathrm{~s}^{-1} \mathrm{sr}^{-1}$ for energies of electrons $10-1000 \mathrm{GeV}$ In principle, it can be taken into account that near the GC the cosmic ray flux increases; the amplifying effect can be simulated by multiplicative factor $f(r(s, \theta))=\exp \left(r_{\odot}-r\right) / r_{0}$, in more detail this factor and called astrophysical factor are describe in[14] and references therein. Here, the DM distribution is modeled by spherically symmetric Einasto profile[16-18].

After integration of (8), there arises the flux of neutrino, $d N / d E_{v}$ spectrum is shown in Fig. 3 and it is most steep in the range $(50-350) \mathrm{GeV}$. Then, integrating $d N / d E_{v}$ over the neutrino energy, the number of neutrino events in the IceCube Neutrino Observatory[19, 20] can be estimated. Namely, for the half hemisphere solid angle and the IceTop surface area, $A=10^{6} \mathrm{~m}^{2}$, we have a small number of landing neutrino - even taking into account the flux amplifying factor $f$, we have $N_{v}=(6-7)$ events per year; this number depends on the used DM profile very slightly. Note, the possibility to detect these events at IceCube is very small[6], moreover, there is an atmospheric neutrino bkg which is large at these energies[21, 22].

The second DM component, H-baryons, interact with "ordinary world" via scalars, Higgs and $\mathrm{H}-\sigma, \mathrm{H}$-pion and $\mathrm{H}$-quark loops do not contribute to the scattering amplitude in analogy with Furrry's theorem. So, we consider inclusive process $e B \rightarrow v_{e} W^{-} B$ (or $\bar{B}$ ); note, reactions like $l^{-} B \rightarrow v_{l} W^{-} B$ and $v_{e} B \rightarrow e^{-} W^{+} B$ are analyzed in the same way. Corresponding diagram is shown in Fig. 4. 


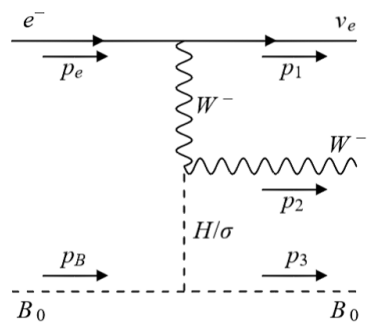

Figure 4. Lepton scattering via scalars off the B-component of the Dark Matter.

This process is more cumbersome for the analysis, and the cross section can be presented in the double-differential form as:

$$
\begin{gathered}
d \sigma=C \int \frac{I_{2}\left(t_{1}, s_{2}\right)}{\left(t_{1}-m_{W}^{2}\right)^{2}} d t_{1} d s_{2}, \\
I_{2}=C_{H}^{2} I_{H}+C_{\sigma}^{2} I_{\sigma}+2 C_{H} C_{\sigma} I_{H \sigma},
\end{gathered}
$$

where $I_{H}, I_{\sigma}$ and $I_{H \sigma}-$ are integrals of the type:

$$
\begin{gathered}
I_{H}=\left[D_{1}\left(t_{2}-m_{H}^{2}\right)+2\left(E_{1}+2 m_{H}^{2} D_{1}\right) \ln \left(t_{2}-m_{H}^{2}\right)-\right. \\
\left.-\left(F_{1}+m_{H}^{4} D_{1}+m_{H}^{2} E_{1}\right) \frac{1}{t_{2}-m_{H}^{2}}\right]\left.\right|_{t_{2}^{-}} ^{t_{2}^{+}} \\
I_{H \sigma}=\frac{D_{1}}{2}\left(t_{2}^{+}-t_{2}^{-}\right)\left(m_{H}^{2}+m_{\sigma}^{2}\right)+\frac{1}{2}\left(E_{1}-D_{1}\left(m_{H}^{2}+m_{\sigma}^{2}\right)\right) \times \\
\times\left.\ln \left[\left(t_{2}-\frac{1}{2}\left(m_{H}^{2}+m_{\sigma}^{2}\right)\right)^{2}+\frac{1}{4}\left(m_{\sigma}^{2}-m_{H}^{2}\right)^{2}\right]\right|_{t_{2}^{-}} ^{t_{2}^{+}}+ \\
+\left.\frac{2 F_{1}+2 m_{H}^{2} m_{\sigma}^{2} D_{1}+E_{1}\left(m_{H}^{2}+m_{\sigma}^{2}\right)}{m_{\sigma}^{2}-m_{H}^{2}} \arctan \left[\frac{t_{2}-m_{\sigma}^{2}-m_{H}^{2}}{m_{\sigma}^{2}-m_{H}^{2}}\right]\right|_{t_{2}^{-}} ^{t_{2}^{+}}, \\
I_{\sigma}=I_{H}\left(m_{H} \rightarrow m_{\sigma}\right),
\end{gathered}
$$

Here, limits of integration are:

$$
\begin{gathered}
t_{2}^{ \pm}=2 m_{B}^{2}-\frac{1}{2 s_{2}}\left[\left(s_{2}+m_{B}^{2}-t_{1}\right)\left(s_{2}+m_{B}^{2}-q^{2}\right) \mp\right. \\
\left.\mp \lambda^{1 / 2}\left(s_{2}, m_{B}^{2}, t_{1}\right) \lambda^{1 / 2}\left(s_{2}, q^{2}, m_{B}^{2}\right)\right]
\end{gathered}
$$

and vertex factors for two diagrams are the following: $C_{H}=c_{\theta}\left(\lambda_{01} v c_{\theta}+2 \lambda_{11} u s_{\theta}\right), C_{\sigma}=$ $s_{\theta}\left(\lambda_{01} v s_{\theta}-2 \lambda_{11} u c_{\theta}\right)$. Now, analogously to previous reaction, we calculate differential and total cross section presenting them for some parameter values. In Fig. 5 and 6 cross section in dependence on neutrino energy is shown for exclusive reaction $e B \rightarrow v_{e} W^{-} B \rightarrow v_{e} l^{-} v_{l} B$. Indeed, inclusive cross section is larger in nine times, approximately, as it results from narrow width approximation for $\mathrm{W}$ decay. Total cross section strongly depends on H-baryon mass as it can be seen in Fig.7. Then, the cross section can be integrated with cosmic electron spectrum, as for the scattering off H-pions. Taking into account the DM distribution and the IceCube parameters, as above, we estimate the number of neutrino landings as 6-7. So, totally, 
the scattering of cosmic electrons with energies up to $1 \mathrm{TeV}$ does not produce a significant neutrino signal at IceCube. At the same time, this process can result to some visible manifestation of dense clusters of hidden mass, clumps[23-29]. If they are, the number of neutrino events can be increased in 2-5 times due to proportionality to the clump density[30, 31].

Note, the same cross section for exclusive reaction of neutrino scattering can result to positron production due to $W^{+}$decay, positron energies should be mostly in the interval $\approx$ $(200-400) \mathrm{GeV}$.

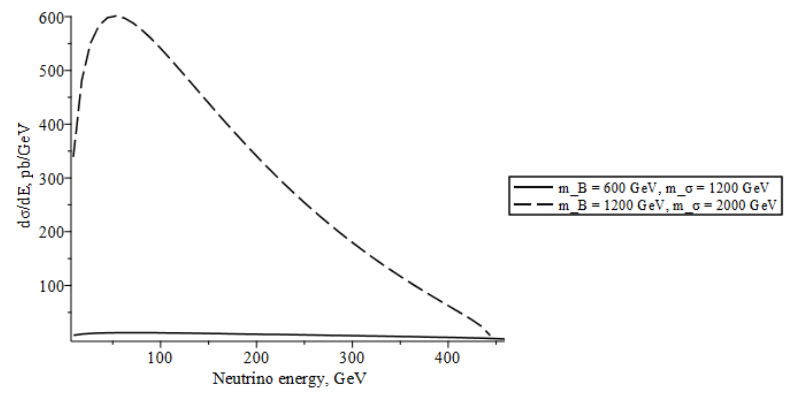

Figure 5. Cross section of exclusive lepton scattering off H-baryons in dependence of secondary lepton energy; energy of initial lepton $500 \mathrm{GeV}$.

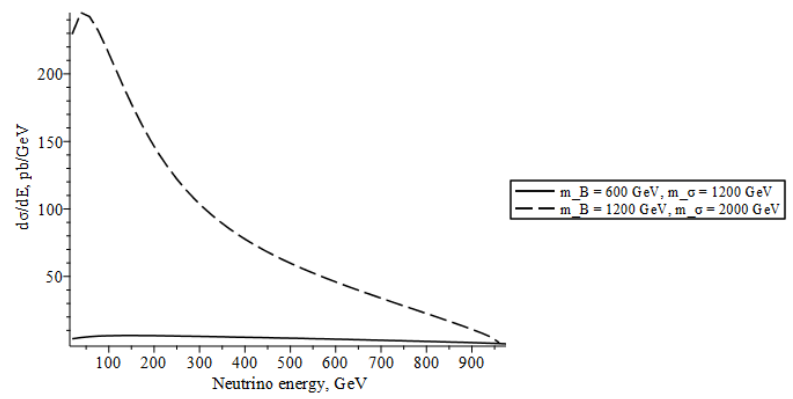

Figure 6. Cross section of exclusive lepton scattering off H-baryons in dependence of secondary lepton energy; energy of initial lepton $1000 \mathrm{GeV}$.

\section{Conclusion}

It is suggested to consider the scattering of high-energy cosmic rays as an additional way to study the nature and distribution of hidden mass. In the model with additional heavy fermions-hyper-quarks - there appear two different pNG states which are neutral and stable. So, they can be interpreted as heavy DM candidates. from analysis of kinetics of their burning out it follows estimation of masses of these states assuming their masses are close.

To analyze the scattering of cosmic electrons off $\mathrm{H}$-pions and $\mathrm{H}$-baryons, differential and total cross sections were calculated together with secondary neutrino fluxes. The number of neutrino landings to the IceCube surface were also estimated, these values are small in comparison with the background of atmospheric neutrinos at the IceCube. 


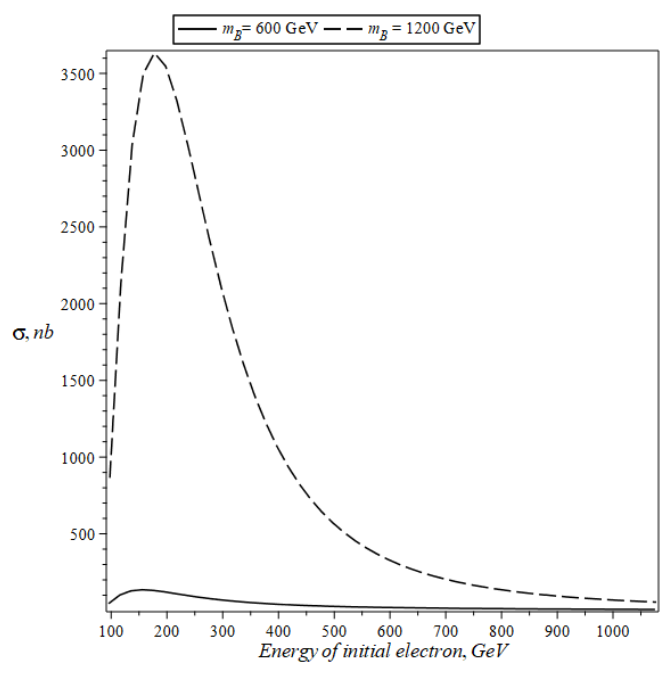

Figure 7. Total scattering cross section depending on initial electron energy for two values of H-baryon mass.

However, the scattering of cosmic rays off the DM clumps can lead to substantial increasing of secondary neutrino flux and the number of events. These compact regions of high DM density can increase the secondary neutrino flux in 2-5 times depending on the DM density. Note, annihilation signals from the clumps can be also noticeably larger[29]. There is, however another pessimistic factor - a sharp decreasing of the cosmic ray electron spectrum with energy. Besides, the fraction of cosmic electrons is small in a total cosmic ray flux density. Nevertheless, this type of analysis can be effective for the studying of the DM space distribution using the data on neutrino spectrum with energies $\lesssim 1 \mathrm{TeV}$; to realize this it is necessary to have some space map of neutrino spectra depending on various directions. It should be an important task for neutrino observatories and space telescopes.

Scattering of galactic and extra-galactic neutrino off the DM is another interesting channel that can hive rise in positrons (together with other leptons) yield due to decay of secondary $W^{+}$; these exclusive cross sections were shown in Section 4. Certainly, the scattering of protons off scalar DM will also contribute to the positrons production from W-boson decay, because of much larger flux of cosmic protons than electrons, cross sections of such inclusive reaction can be approximately up to an order larger.

So, an analysis of spectra of neutrino and leptons from high-energy cosmic rays interaction with components of the DM can give a valuable information on the hidden mass origin and its distribution in space. Besides, it seems, in all models where the DM candidates interact with leptons, quarks and vector bosons only via Higgs boson and some possible additional scalars ( $\tilde{\sigma}-$ meson, for example) the hidden mass should manifests itself via analogous signals with model dependent intensities.

Acknowledgments. This work is supported by Russian Scientific Foundation (RSCF) Grant N 18-12-00213.

\section{References}

[1] C. Kilic, T. Okui, R. Sundrum, JHEP 1002, 033 (2015). 
[2] R. Pasechnik, V. Beylin, V. Kuksa, G. Vereshkov, Phys. Rev. D88, 075009 (2013).

[3] V. A. Beylin, G. M. Vereshkov, V. I. Kuksa, Phys. Part. Nucl. Lett. 13, 1 (2016).

[4] Y. Bai and R. J. Hill, Phys. Rev. D 82, 111701 (2010).

[5] V. Beylin, M. Bezuglov, V. Kuksa, N. Volchanskiy, Adv. in HEP. 2017, 1765340 (2017).

[6] V. Beylin, M. Bezuglov, V. Kuksa, E. Tretyakov, A. Yagozinskaya, Int.J.of Mod. Phys. A 34, 6(7) (2019). arXiv:hep-ph/1810.00372.

[7] R. Pasechnik, V. Beylin, V. Kuksa, G. Vereshkov, Eur. Phys. J. C 74, 08 (2013).

[8] T. A. Ryttov, F. Sannino, Phys. Rev. D 78, 115010 (2008). arXiv:0809.0713 [hep-ph].

[9] V. Beylin, M. Bezuglov, and V. Kuksa, Int. J. Mod. Phys. A 32, 1750042 (2017).

[10] K. Griest and D. Seckel, Phys. Lett. B 43, 3191 (1991).

[11] XENON Collab. (E. Aprile et al.) Phys. Rev. Lett. 119, 181301 (2017).

[12] DARWIN Collab. (J. Aalbers et al.) JCAP 1611, 017 (2016).

[13] E. Boos, I. Volobuev, Phys. Rev. D 97, 095014 (2018).

[14] S. Profumo and L. Ubaldi, JCAP 020, 1108 (2011). arXiv:1106.4568 [hep-ph]

[15] CALET Collab. (O. Adriani et al), Phys. Rev. Lett. 119, 181101 (2017). arXiv:1712.01711.

[16] M. Cirelli, J. Phys.: Conf. Ser. 447, 012006 (2013). preprint: SACLAY-T13/021.

[17] M. Cirelli, arXiv:1202.1454 [astro-ph] (2012).

[18] A. A. Dutton, A. V. Maccio, Mon. Not. R. Astron. Soc. 441, 4 (2014). arXiv:1402.7073v2 [astro-ph].

[19] IceCube Collab. (A. Ishihara et al), J. Phys.: Conf. Ser. 718, 062027 (2016).

[20] IceCube Collab. (M. G. Aartsen et al), Science 342, 1242856 (2013). arXiv:1311.5238 [astro-ph].

[21] IceCube Collab. (R. Abbasi et al), arXiv:1108.0171v2 [astro-ph] (2011).

[22] IceCube Collab. (J. Ahrens et al), Astropart.Phys. 20,507 (2004). arXiv:astro$\mathrm{ph} / 0305196$.

[23] V. Berezinsky, V. Dokuchaev, Y. Eroshenko, Phys. Rev. D 68, 103003 (2003). arXiv: 0301551 [astro-ph].

[24] V. Berezinsky, V. Dokuchaev, Y. Eroshenko, Phys. Rev. D 77, 083519 (2008). arXiv:0712.3499 [hep-ph].

[25] V. Berezinsky, V. Dokuchaev, Y. Eroshenko, JCAP 059, 1311 (2013). arXiv:1308.6742 [hep-ph].

[26] V. Berezinsky, V. Dokuchaev, Y. Eroshenko, Phys. Usp. 57, 1 (2014). arXiv:1405.2204 [hep-ph].

[27] D. Erkal, S. Koposov, V. Belokurov, arXiv:1609.01282 [astro-ph] (2016).

[28] K. Belotsky, A. Kirillov, M. Khlopov, Grav. Cosmol. 20, 47 (2014). arXiv:1212.6087 [astro-ph].

[29] K. Belotsky, A. Kirillov, M. Khlopov, Adv. in HEP 2014, 651247 (2014). arXiv:1312.6853.

[30] A. Tasitsiomi and A. V. Olinto, Phys. Rev. D 66, 083006 (2002).

[31] R. Aloisio, P. Blasi, A. V. Olinto, Astrophys. J. 60147 (2004). arXiv:0206036 [astro$\mathrm{ph}]$. 